Received: 27 March 2018

Accepted: 25 October 2018

Published online: 06 November 2018

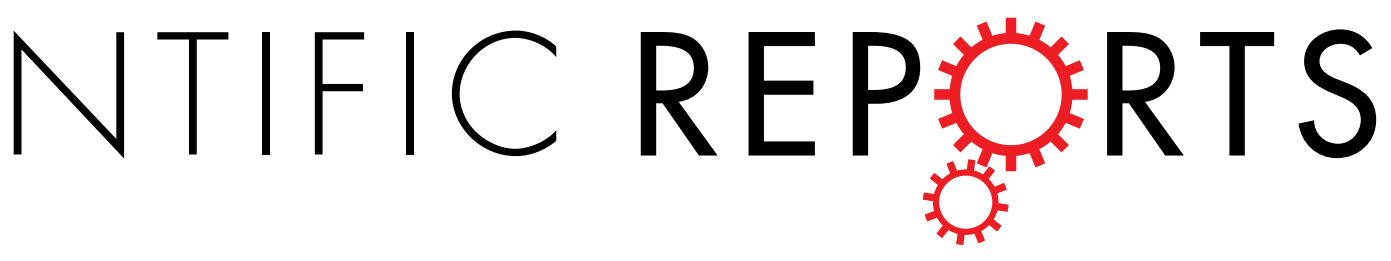

\title{
OPEN The effects of in ovo administration of encapsulated Toll-like receptor 21 ligand as an adjuvant with Marek's disease vaccine
}

\author{
Jegarubee Bavananthasivam ${ }^{1}$, Leah Read ${ }^{1}$, Jake Astill ${ }^{1}$, AlexanderYitbarek ${ }^{1}$, \\ Tamiru N. Alkie ${ }^{1,2}$, Mohamed Faizal Abdul-Careem ${ }^{3}$, Sarah K. Wootton $\mathbb{1}^{1}$, \\ Shahriar Behboudi ${ }^{4,5}$ \& Shayan Sharif ${ }^{1}$
}

Marek's Disease Virus (MDV) is the causative agent of a lymphoproliferative disease, Marek's disease (MD) in chickens. MD is only controlled by mass vaccination; however, immunity induced by MD vaccines is unable to prevent MDV replication and transmission. The herpesvirus of turkey (HVT) vaccine is one of the most widely used MD vaccines in poultry industry. Vaccines can be adjuvanted with Tolllike receptor ligands (TLR-Ls) to enhance their efficacy. In this study, we examined whether combining TLR-Ls with HVT can boost host immunity against MD and improve its efficacy. Results demonstrated that HVT alone or HVT combined with encapsulated CpG-ODN partially protected chickens from tumor incidence and reduced virus replication compared to the control group. However, encapsulated CpGODN only moderately, but not significantly, improved HVT efficacy and reduced tumor incidence from $53 \%$ to $33 \%$. Further investigation of cytokine gene profiles in spleen and bursa of Fabricius revealed an inverse association between interleukin (IL)-10 and IL-18 expression and protection conferred by different treatments. In addition, the results of this study raise the possibility that interferon (IFN)- $\beta$ and IFN- $\gamma$ induced by the treatments may exert anti-viral responses against MDV replication in the bursa of Fabricius at early stage of MDV infection in chickens.

Marek's disease (MD) in chickens is caused by an alpha herpesvirus, Marek's Disease Virus (MDV), which is currently controlled in chicken farms by vaccination, selection of genetic lines resistant to MD and biosecurity measures. Depending on the host and virus virulence, MDV can cause more than $90 \%$ morbidity and mortality in susceptible chickens ${ }^{1}$. Chickens become infected by MDV following inhalation of infected dust via the respiratory route. MDV primarily infects macrophages, $\mathrm{B}$ and $\mathrm{T}$ cells and it mainly transforms CD4+ T cells although other T cell subtypes are susceptible to transformation ${ }^{2,3}$. Generally, MDV life cycle can be divided into the cytolytic phase (2-7 days post-infection - dpi), latent phase (7-10 dpi), late cytolytic phase, and transformation phase ${ }^{2}$. Lysis of lymphocytes and activation of TGF-beta + regulatory T cells are a few mechanisms among others which induce immunosuppression during MDV infection ${ }^{4}$. Furthermore, transformed $\mathrm{T}$ cells proliferate form lymphoma which can lead to immunosuppression and clinical and pathological signs of disease. In the course of infection, MDV replicates in feather follicular epithelium and sheds into the environment throughout the lifespan of an infected chicken.

Since MDV was first identified more than 50 years ago, several vaccines have been developed to control clinical signs of the disease, although none of them can fully prevent replication or transmission of MDV. Because of the inability of vaccines to control MDV transmission, it is believed that vaccines have prompted the emergence of virulent strains of $\mathrm{MDV}^{5}$. Herpesvirus of turkey (HVT) is one of the MD vaccines which is extensively used worldwide either alone or in combination with other MD vaccine ${ }^{6}$. HVT can be administered in ovo at embryonic

\footnotetext{
${ }^{1}$ Department of Pathobiology, Ontario Veterinary College, University of Guelph, Guelph, Ontario, N1G 2W1, Canada. ${ }^{2}$ Present address: Department of Biology, Wilfred Laurier University, Waterloo, Ontario, N2L 3C5, Canada. ${ }^{3}$ Department of Ecosystem and public health, Faculty of Veterinary Medicine, University of Calgary, Calgary, Alberta, T2N 1N4, Canada. ${ }^{4}$ The Pirbright Institute, Ash Road, Pirbright, Woking, Surrey, GU24 0NF, UK. ${ }^{5}$ Department of Pathology and Infectious Disease, School of Veterinary Medicine, University of Surrey, Guildford, United Kingdom. Correspondence and requests for materials should be addressed to S.S. (email: shayan@uoguelph.ca)
} 


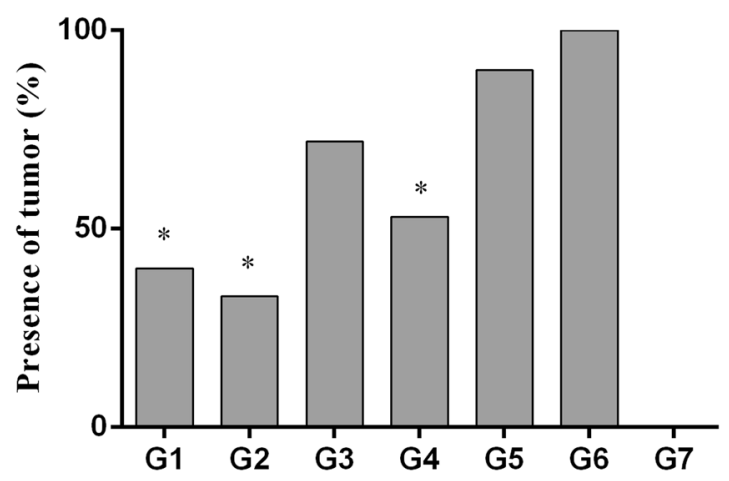

Figure 1. Presence of tumors in different treatment groups. Experimental groups were as follows: G1-ECpG and HVT were administered at ED18 and the second dose of ECpG was injected at 14 dpi. G2-ECpG and HVT were administered at ED18. G3-HVT was administered at ED18 and ECpG was given at 14 dpi. G4-HVT was administered at ED18. G5-ECpG was injected at 14 dpi. G6-Untreated, MDV-infected group. G7-PBS control group. Chickens in all groups were infected with MDV at d5 except G7. Presence of tumors was recorded at 21 dpi. Data were statistically analyzed by Fisher's exact test in GraphPad Prism 6.04. $p \leq 0.05(*)$ was considered statistically significant compared to G6.

day 18 (ED18) to provide protection against MDV in young chicks. However, protection offered by HVT is inadequate especially against very virulent and very virulent plus pathotypes of $\mathrm{MDV}^{7}$. Moreover, this vaccine will not be protective against emerging highly virulent strains of MDV in the future. Therefore, it would be of interest to determine whether efficacy of HVT can be enhanced by addition of immune stimulants.

Innate and adaptive defense mechanisms are necessary to control MDV infection in chickens. In young chicks, it is likely that in ovo administered vaccines initially confer protection mainly via innate immune system cells including Natural Killer (NK) like cells and macrophages rather than adaptive immune responses ${ }^{8-11}$. In general, the activation of innate responses orchestrates the induction of adaptive responses which in turn require several days to provide protective immunity against an infection. However, this is not well understood in regard to MD vaccination. In general, the immune system in young chicks undergoes maturation and becomes functionally mature around 1-2 weeks of age. Therefore, the induction of adaptive immune responses might be limited in the early stage of life and immediately after hatching. It has been reported that chicken T cells exhibit poor responsiveness to mitogen stimulation and are functionally immature during the first 2 weeks of age ${ }^{12}$. In addition, although it has been shown that chickens start to mount antibody-mediated immune responses at 1 week of age, they usually display higher levels of antibody production when they are immunized around 2 weeks of age $e^{13,14}$. There is a paucity of information on how MD vaccine modulates innate responses in young chickens. The constitutive expression of IFN- $\gamma$ and IL-18 that is measurable from ED12 and higher expression of these cytokines by day 7 post-hatch ${ }^{15}$ indicates that innate responses may be functional prior to hatching. As a result, these responses can be exploited to accelerate the maturation of innate and adaptive immune responses. Increased IFN- $\gamma$ expression observed with in ovo HVT vaccination in chickens ${ }^{16}$ as well as increased NK like activity observed with HVT vaccination at hatch and post-hatch ${ }^{17,18}$ revealed that innate responses can be elevated to significant level with appropriate immune modulations ${ }^{19}$. In addition, it has recently been reported that in ovo administration of HVT vaccine accelerates the maturation of the immune system by activation and expansion of $\mathrm{T}$ cells at hatch ${ }^{20}$.

TLR-Ls have been used experimentally as vaccine adjuvants or as stand-alone anti-viral agents ${ }^{21-24}$. For instance, TLR3 ligand, polyinosinic:polycytidylic acid (Poly(IC)), has been shown to induce innate responses in chickens ${ }^{25}$ and its administration to chickens combined with HVT could lead to reduction of tumor incidence after challenge with a very virulent strain of $\mathrm{MDV}^{26}$. In addition, TLR4 ligand, lipopolysaccharide (LPS), and CpG-ODN, the ligand for TLR2 1 have been shown to stimulate innate responses mainly via the induction of interferons (IFNs) and interleukin (IL) $1-\beta$ and, which may lead to a reduction in MD progression in chickens $^{27}$. Prolonged availability of TLR-Ls in a controlled-release fashion which can be achieved by encapsulation in poly(D, L-lactic-co-glycolic) acid (PLGA) nanoparticles has been shown to induce innate responses in vitro, in ovo and in vivo ${ }^{19,28}$. Administration of encapsulated $\mathrm{CpG}(\mathrm{ECpG})$ as a prophylactic agent demonstrated considerable effects on the outcome of MD in our previous study ${ }^{29}$, which prompted further investigation of the adjuvant effect of ECpG with MDV vaccine in chickens.

The present study was designed to study the adjuvant effect of ECpG in conjunction with HVT vaccine administered either in ovo to ED18 embryos or to chickens 14 days after an experimental infection with MDV or at both time points to improve immunity against MDV infection in chickens.

\section{Results}

MDV tumor incidence. Treatment groups were compared to the positive control group (G6), in which all chickens developed tumors (Fig. 1, Supplementary Table 1). Chickens in group 1 that received ECpG and HVT at ED18, and ECpG again at 14 dpi showed $40 \%$ tumors which was statistically significant compared to positive control chickens $(p=0.002$, Fig. 1). Chickens in group 2 which received ECpG and HVT at ED18 developed the lowest tumor incidence compared to positive control chickens $(33.33 \%, p=0.001)$. In HVT only group (G4), $53.33 \%$ of the chickens exhibited tumors, which was significantly different compared to positive control chickens 


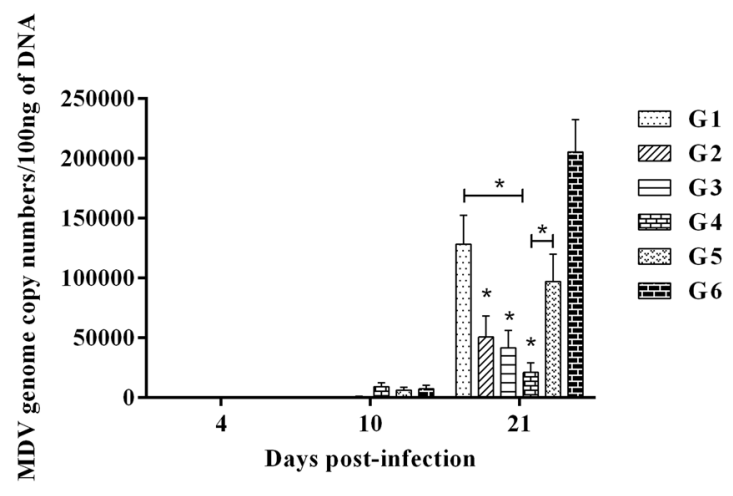

Figure 2. MDV genome copy numbers in feathers in different treatment groups. MDV genome copy numbers per $100 \mathrm{ng}$ of DNA were calculated from feathers collected at 4,10 and $21 \mathrm{dpi}$. The different experimental groups were: G1-ECpG and HVT were administered at ED18 and the second dose of ECpG was injected at 14 dpi. G2ECpG and HVT were administered at ED18. G3-HVT was administered at ED18 and ECpG was given at 14 dpi. G4-HVT was administered at ED18. G5-ECpG was injected at 14 dpi. G6-Untreated, MDV-infected group. G7-PBS control group. Chickens in all groups were infected with MDV at d5 except G7. Data were statistically analyzed by Kruskal-Wallis test in GraphPad Prism 6.04. $p \leq 0.05$ was considered statistically significant $(*)$. Comparisons were made between groups as indicated or with positive control group G6. Error bars indicate the standard errors of the mean.
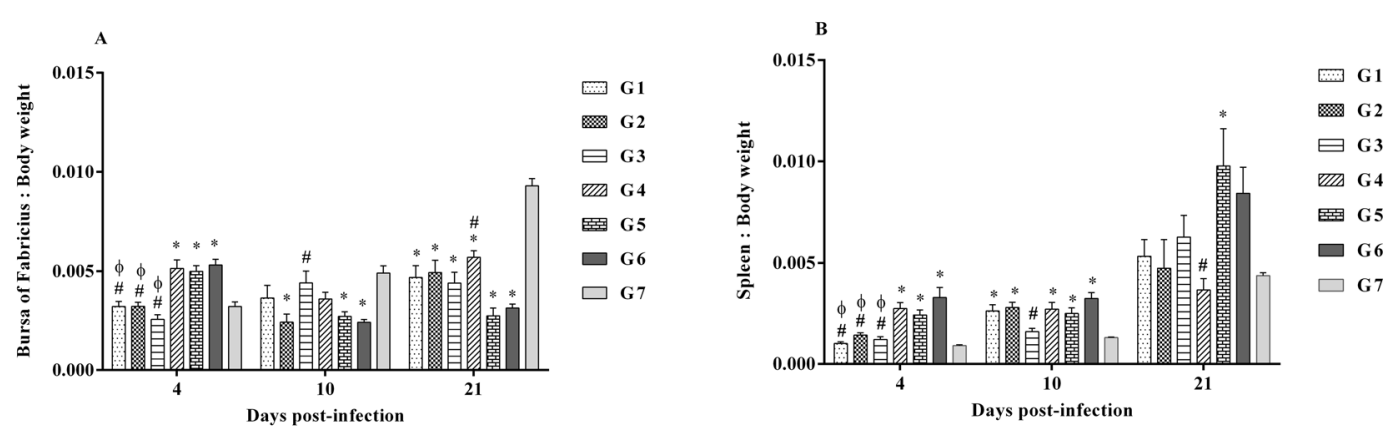

Figure 3. BF:BW and spleen:BW ratios in different treatment groups. BF, spleen and body weight were recorded at 4, 10 and $21 \mathrm{dpi}$, and (A) BF:BW and (B) Spleen:BW ratios were calculated. The different experimental groups were: G1-ECpG and HVT were administered at ED18 and the second dose of ECpG was injected at 14 dpi. G2-ECpG and HVT were administered at ED18. G3-HVT was administered at ED18 and ECpG was given at 14 dpi. G4-HVT was administered at ED18. G5-ECpG was injected at 14 dpi. G6-Untreated, MDV-infected group. G7-PBS control group. Chickens in all groups were infected with MDV at d5 except G7. Data were statistically analyzed by one-way ANOVA and followed by Tukey's multiple comparison test in GraphPad Prism 6.04. $p \leq 0.05$ was considered statistically significant when compared to G7 (*) or G6 (\#) or G4 $(\phi)$. Error bars indicate the standard errors of the mean.

( $p=0.01$, Fig. 1). In G3 and G5, $72.72 \%$ and $90 \%$ of the chickens developed tumors respectively (Fig. 1 ). There was no statistically significant difference in tumor incidence between chickens in G4 and ECpG administered groups (G1, G2 and G3). However, the highest reduction in tumor incidence was observed in chickens in G2 (Fig. 1). Therefore, it seems that ECpG partially improved the HVT induced protection against MD.

MDV genome copy numbers in feathers. At 4 dpi, there was no genome detected in feather DNA. MDV load gradually increased throughout the experiment and was at the highest level at $21 \mathrm{dpi}$ (Fig. 2). Chickens in group 2, 3 and 4 had significantly lower MDV load in feathers at 21 dpi when compared to that of G6 $(p<0.0001$, Fig. 2). In addition, significantly lower MDV load was detected in G4 chickens when compared to G1 and G5 chickens $(p<0.0001$, Fig. 2). Unfortunately, administration of ECpG was unable to enhance the HVT induced reduction of MDV load in feathers.

Organ weight indices. At $4 \mathrm{dpi}$, bursa of Fabricius weight to body weight (BF:BW) ratios were significantly higher in G4, G5 and G6 chickens when compared to the chickens in the PBS control group (G7) $(p<0.0001$, Fig. 3A). Although at 4 dpi BF:BW ratios in G1, G2 and G3 chickens were almost similar when compared to G7 chickens, they were significantly different from that of G6 chickens ( $p<0.0001$, Fig. 3A). At 10 dpi, G2, G5 and G6 chickens showed significantly lower BF:BW ratios when compared to G7 chickens $(p=0.0001)$. However, G3 chickens exhibited significantly higher ratios when compared to G6 chickens ( $p=0.0001$, Fig. 3A). At 21 dpi, chickens in all MDV-infected groups showed significantly lower BF:BW ratios when compared to G7 chickens 

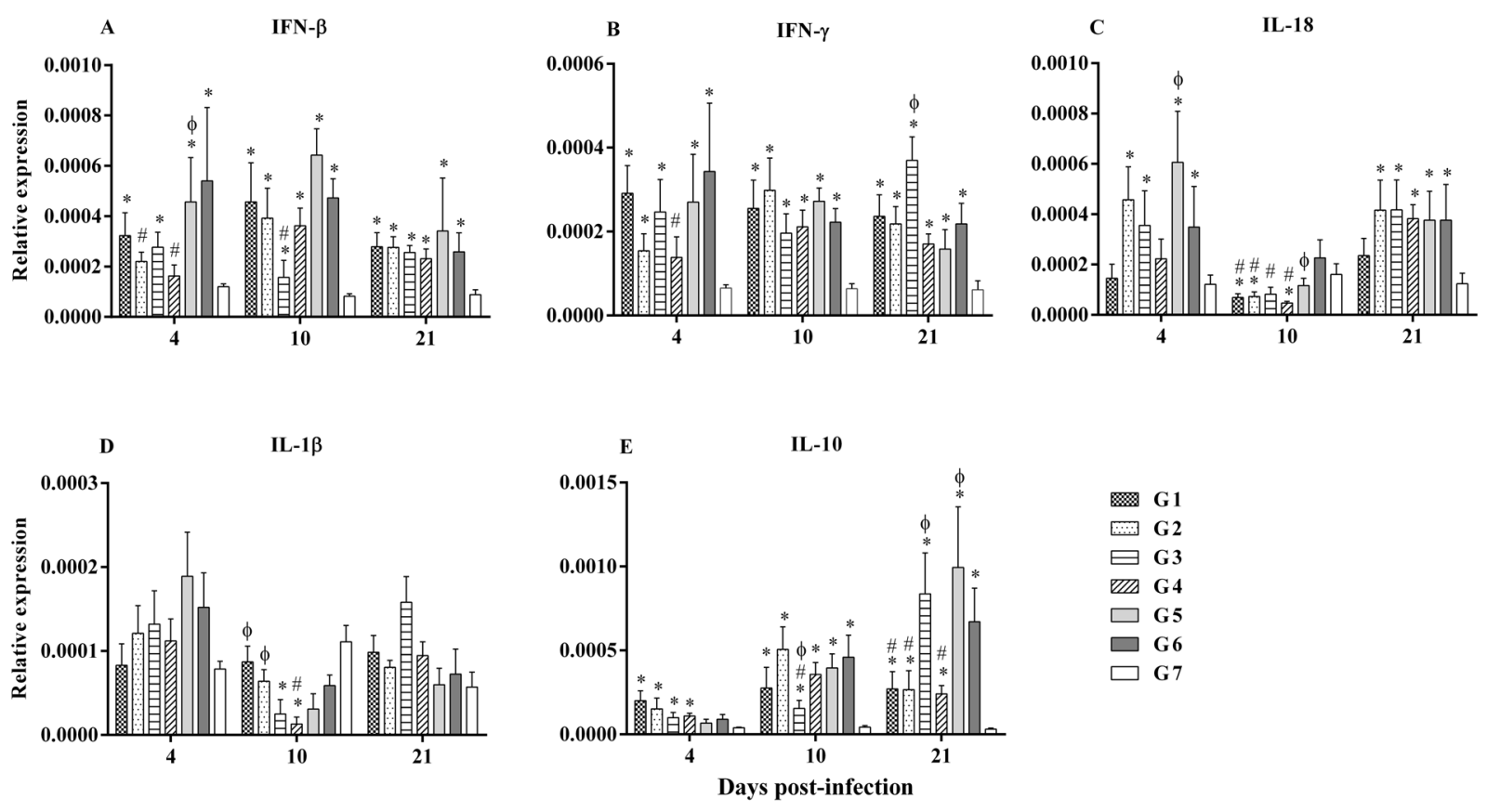

Figure 4. Relative expression of genes in spleen in different treatment groups. (A) IFN- $\beta$ (B) IFN- $\gamma$ (C) IL-18 (D) IL-1 $\beta$ and (E) IL-10 expression were determined relative to $\beta$-actin at 4,10 and 21 dpi in spleen. The different experimental groups were: G1-ECpG and HVT were administered at ED18 and the second dose of ECpG was injected at 14 dpi. G2-ECpG and HVT were administered at ED18. G3-HVT was administered at ED18 and ECpG was given at 14 dpi. G4-HVT was administered at ED18. G5-ECpG was injected at 14 dpi. G6-Untreated, MDV-infected group. G7-PBS control group. Chickens in all groups were infected with MDV at d5 except G7. Data were logarithmically transformed and analyzed using general linear model (Proc GLM) and followed by Duncan's multiple range test in Statistical Analysis Software version 9.3 (SAS, Cary, NC). The Kruskal-Wallis test was used when data were not normally distributed. Gene expression results were presented as geometric mean of relative expression \pm standard error of mean. $p \leq 0.05$ was considered statistically significant when compared to G7 $(*)$ or G6 (\#) or G4 $(\phi)$.

$(p<0.0001$, Fig. 3A). However, the ratio was significantly higher in G4 when compared to G6 $(p<0.0001$, Fig. 3A). This indicates that HVT vaccine can partially inhibit bursal atrophy induced by MDV.

At 4 dpi, spleen:BW ratios were significantly higher in G4, G5 and G6 chickens when compared to G7 chickens and ratios were significantly lower in G1, G2 and G3 chickens when compared to G6 chickens $(p<0.0001$, Fig. 3B). At $10 \mathrm{dpi}$, chickens in all groups which received MDV except G3 had significantly higher spleen:BW ratios when compared to G7, and G3 chickens had significantly lower spleen:BW ratios when compared to G6 chickens $(p<0.0001$, Fig. 3B). At 21 dpi, G5 chickens showed significantly higher spleen:BW ratio when compared to G7 chickens ( $p<0.0015$, Fig. 3B). However, G4 chickens showed significantly lower ratio when compared to G6 chickens ( $p<0.0015$, Fig. 3B).

Cytokine gene expression. In spleen, IFN- $\beta$ was significantly upregulated at all time points in all MDV-infected groups except G2 and G4 at 4 dpi when compared to G7 ( $p \leq 0.001$, Fig. 4A). Similarly, IFN- $\gamma$ was significantly upregulated at 4, 10 and 21 dpi in all MDV-infected groups, except G4 at 4 dpi, compared to G7 ( $p \leq 0.001$, Fig. 4B). IL-18 was significantly upregulated in G2, G3, G5 and G6 at 4 dpi, whereas this cytokine was downregulated in G1, G2 and G4 at 10 dpi. Again, at 21 dpi, IL-18 was upregulated in G2, G3, G4, G5 and G6 when compared to G7 ( $p \leq 0.025$, Fig. $4 \mathrm{C}$ ). Although there was no significant upregulation of IL-1 $\beta$ at any time point, slightly higher expression of IL- $1 \beta$ was observed in G1, G3 and G4 chickens at 21 dpi when compared to G6 (Fig. 4D). IL-10 expression was significantly upregulated in the chickens in all the groups that received HVT at $4 \mathrm{dpi}(P=0.007)$ as well as in MD-infected groups at 10 and $21 \mathrm{dpi}(p<0.0001)$ when compared to G7 chickens (Fig. 4E). Noticeably, IL-10 expression was significantly lower in G3 chickens at 10 dpi and G1, G2 and G4 chickens at 21 dpi when compared to the chickens in G6 ( $p<0.0001$, Fig. $4 \mathrm{E})$.

In the bursa of Fabricius, at $4 \mathrm{dpi}$, IFN- $\beta$ was significantly upregulated in G1, G3, G4 and G5 chickens when compared to G6 and G7 chickens ( $p<0.0001$, Fig. 5A). At 10 dpi, IFN- $\beta$ was significantly upregulated in G1 chickens when compared to G6 and G7 chickens, and in G2 when compared to G7 ( $p=0.002$, Fig. 5A). At 21 dpi, IFN- $\beta$ was upregulated in all groups that were challenged with $\operatorname{MDV}(p<0.0001$, Fig. 5A). Further, IFN- $\gamma$ was upregulated in chickens at all time points and in all the groups which received MDV when compared to chickens in G7 except the chickens in G2, G5 and G6 at 4 dpi ( $p \leq 0.0002$, Fig. 5B). Expression of IFN- $\gamma$ was significantly upregulated in G1 and G4 chickens at $4 \mathrm{dpi}$ when compared to G6 chickens ( $p \leq 0.0002$, Fig. 5B). Downregulation of IL-18 was observed in G3, G4 and G5 chickens at 4 dpi and also at 10 dpi in chicken in all the groups that received HVT when compared to G6 chickens ( $p \leq 0.009$, Fig. 5C). However, at 21 dpi, IL-18 was upregulated 

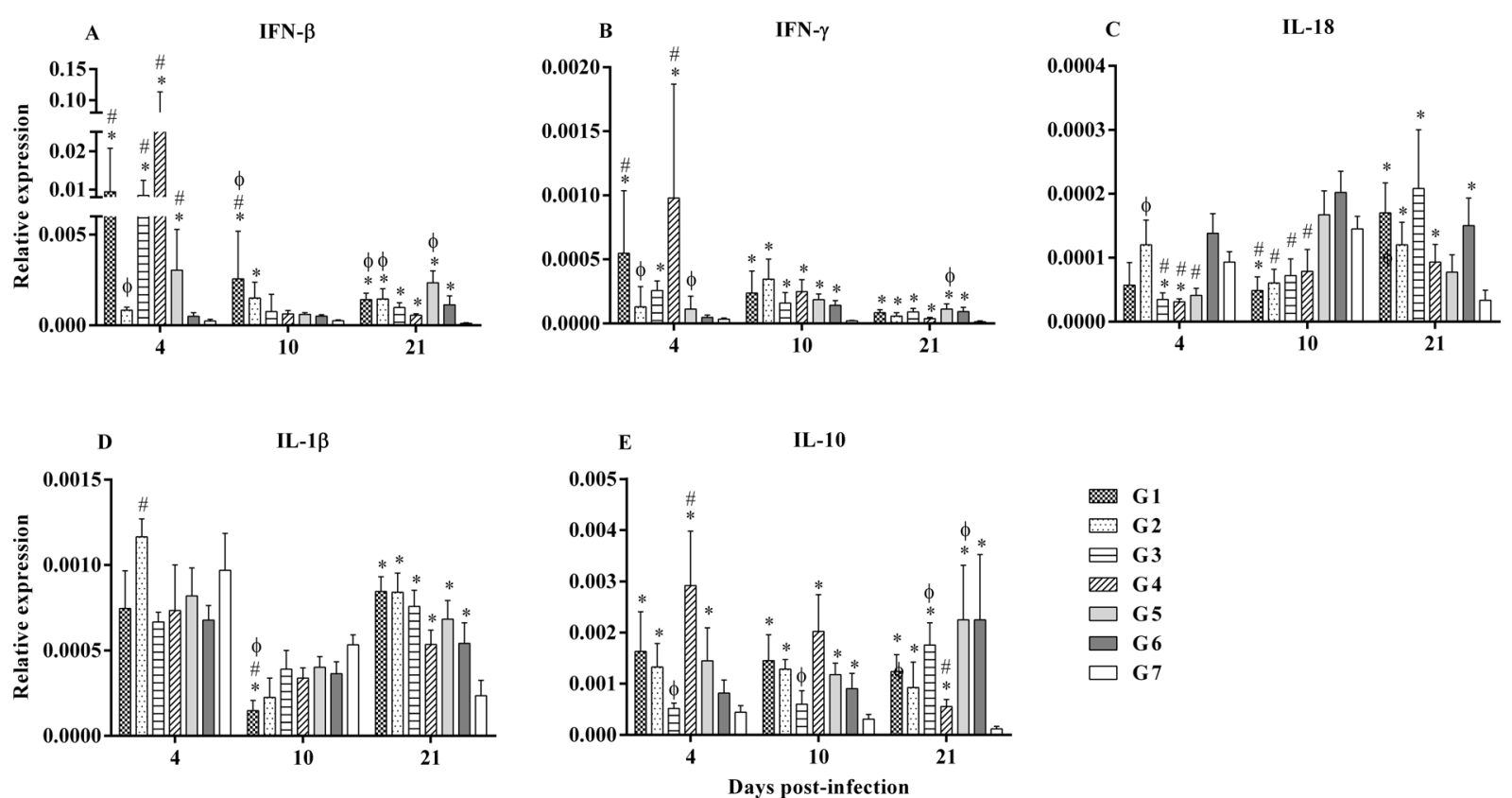

Figure 5. Relative expression of genes in the bursa of Fabricius in different treatment groups. (A) IFN- $\beta$ (B) IFN- $\gamma$ (C) IL-18 (D) IL-1 $\beta$ and (E) IL-10 expression were determined relative to $\beta$-actin at 4, 10 and 21 dpi in BF. The different experimental groups were: G1-ECpG and HVT were administered at ED18 and the second dose of ECpG was injected at 14 dpi. G2-ECpG and HVT were administered at ED18. G3-HVT was administered at ED18 and ECpG was given at 14 dpi. G4-HVT was administered at ED18. G5-ECpG was injected at 14 dpi. G6-Untreated, MDV-infected group. G7-PBS control group. Chickens in all groups were infected with MDV at d5 except G7. Data were logarithmically transformed and analyzed using general linear model (Proc GLM) and followed by Duncan's multiple range test in Statistical Analysis Software version 9.3 (SAS, Cary, NC). The Kruskal-Wallis test was used when data were not normally distributed. Gene expression results were presented as geometric mean of relative expression \pm standard error of mean. $p \leq 0.05$ was considered statistically significant when compared to G7 (*) or G6 (\#) or G4 $(\phi)$.

in chickens in all MDV-infected groups, except in G5 chickens, compared to G7 chickens ( $p=0.001$, Fig. 5C). Expression of IL-1 $\beta$ was upregulated in G2 chickens compared to G6 chickens at 4 dpi (Fig. 5D). IL-1 $\beta$ was upregulated at $10 \mathrm{dpi}$ in $\mathrm{G} 1$ chickens and at $21 \mathrm{dpi}$ in chickens in all the groups that received MDV when compared to G7 chickens ( $p \leq 0.04$, Fig. 5D). Expression of IL-10 was upregulated in the chickens in all MDV-infected group at 4,10 and 21 dpi compared to G7 chickens except G3 and G6 at 4 dpi and, G3 at $10 \mathrm{dpi}(p \leq 0.0005$, Fig. 5E). IL-10 was significantly higher at $4 \mathrm{dpi}$ and lower at $21 \mathrm{dpi}$ in G4 chickens compared to G6 chickens $(p \leq 0.0005$, Fig. 5E).

\section{Discussion}

To enhance host responses against MDV, administration of ECpG along with HVT was investigated in the current study. Among the HVT-administered groups, a significant reduction in both tumor incidence and MDV load in feathers was observed in G2 and G4 chickens. Chickens in G1 showed significant reduction only in tumors whereas chickens in G3 showed significant reduction only in MDV load in feathers. Although there was no statistically significant difference in tumor incidence between chickens in G4 and groups receiving ECpG (G1, G2 and G3), administration of ECpG along with HVT at ED18 appeared to have an additive effect to HVT vaccination, marked by the lowest tumor incidence in $\mathrm{G} 2$ chickens.

The observed reduction of MDV load in feathers of chickens treated with ECpG and HVT or HVT alone is similar to the reduction reported in a previous study using Rispens-CVI988 vaccine ${ }^{30}$, which is known to be the most effective vaccine against very virulent and very virulent plus $M^{3} V^{31}$. Since a reduction of MDV load in feathers was observed in the current study, further investigation of MDV transmission pattern in the environment especially in the dust and between chickens will answer whether this reduction interrupts the spread of MDV. Particularly, the use of resistant and susceptible genotypes of chickens may validate the use of ECpG with HVT as the virulence of MDV can be masked in resistant line of chickens ${ }^{32}$.

Commonly, MDV infection results in bursal atrophy due to B cell cytolysis and spleen enlargement is due to inflammation as well as tumor formation. Hence, organ weight indices were recorded to evaluate the changes occurring in BF and spleen following different treatments and MDV challenge in chickens. Administration of either ECpG and HVT or HVT alone possibly inhibited the initial cytolysis of B cells in BF as indicated by the $\mathrm{BF}$ :BW ratios similar to those of the control group. The observed abrogation of bursal atrophy is similar to the previous reports ${ }^{33,34}$. HVT administration with or without ECpG might have resolved the physiological changes which occurred in spleen at the initial stage of MDV infection since there was no difference in the ratios of 
spleen:BW between the chickens in HVT-administered group and uninfected group at 21 dpi. However, further investigation is needed to test this possibility.

The significant upregulation of the cytokines, IFN- $\beta$ and IFN- $\gamma$, in BF at 4 dpi raises the possibility that the anti-viral activity of these cytokines might have repressed MDV replication at the initial stage of MDV pathogenesis and led to the reduction in tumor incidence and MDV load in feathers. Previous study indicated that involvement of IFN- $\beta$ and IFN- $\gamma$ in preventing DNA and virion synthesis in the case of herpes simplex virus type 1 (HSV1) infection ${ }^{35}$. Since MDV and HSV belong to the subfamily alphaherpesvirinae, we can speculate that IFN- $\beta$ and IFN- $\gamma$ may have similar functions for preventing the replication of MDV in infected cells. Another study also revealed that TLR-Ls induced IFN- $\beta$ expression provide protection against HSV2 infection ${ }^{36}$. These lines of evidence and our findings support the role played by IFN- $\beta$ induced by ECpG and HVT in immunity against MD in chickens. IFN- $\gamma$ possibly exerts anti-viral effects on MDV-infected cells directly or indirectly via activation of macrophages ${ }^{37}$. Significant upregulation of IFN $-\gamma$ at $4 \mathrm{dpi}$ in BF in some treatment groups compared to G6 indicates initial anti-viral effects which might have contributed to the subsequent reduction of tumors in chickens. Overall, the expression profile of IFN- $\gamma$ in spleen and BF shows the association of IFN- $\gamma$ with immunity to MDV infection. This is in agreement with previous studies showing the importance of IFN- $\gamma$ in HVT conferred immunity in chickens ${ }^{38,39}$.

IL-10 expression in chickens is associated with MD progression. It has been suggested that IL-10 curtails T helper (Th) 1 immune responses, which are critical for protection, by deviating responses towards Th2 responses ${ }^{40}$. This could also be the case for MDV infection as an association between IL-10 expression and the disease was reported $^{41,42}$. IL-10 affects dendritic cell (DC) maturation by inhibition of costimulatory molecules, DC induced $\mathrm{T}$ cell activation, antigen-specific T cell proliferation and function ${ }^{43,44}$; and it promotes the expansion of inducible $\mathrm{T}$ regulatory cells $\mathrm{s}^{45}$. Notably, the experimental groups in the current study that exhibited significant reduction in tumor incidence displayed lower expression of IL-10 in spleen at $21 \mathrm{dpi}$. In addition, chickens that had tumors, expressed elevated levels of IL-10 in spleen and BF compared to those chickens that did not have tumors in each treatment group (data not shown). A similar pattern of IL-10 expression was recorded in previous studies in a different context, for example when chickens were vaccinated with bivalent or Rispens-CVI988 vaccine followed by MDV infection ${ }^{30,41}$. Therefore, previous reports were further confirmed and extended in the current study as the suppression of IL-10 was inversely associated with protection.

The other interesting finding in the current study was the downregulation of proinflammatory cytokines, particularly IL-18 expression in chickens in the treatment groups compared to G6 chickens. It has been noted previously that expression of IL-18 diminished in MD resistant lines of chickens ${ }^{46}$ compared to that of susceptible lines and also in vaccine protected chickens compared to unvaccinated-infected controls ${ }^{39,41}$. Similar observations were confirmed in the current study as indicated by the association between decreased expression of IL-18 and protection against MDV as shown by the reduction of tumor incidence and MDV load in feathers. Moreover, IL-18 supports the metastasis of melanoma cells via elevated expression of vascular cell adhesion molecule-1 $(\mathrm{VCAM}-1)^{47}$. Therefore, it is probable that decreased expression of IL-18 in the current study may have abrogated the expression of VCAM-1, which possibly contributed to the reduction of tumor incidence.

In conclusion, administration of ECpG with HVT provided protection against MDV in chickens by reducing tumor incidence and MDV loads in feathers. The anti-viral ability of IFN- $\beta$ and IFN- $\gamma$ might have contributed to the outcome of the treatments against MDV infection. In addition, downregulation of IL-10 and IL-18 by HVT and/or ECpG treatment appears to be linked to defense against MDV infection as demonstrated by the significant reduction of tumor formation and MDV load in feathers.

\section{Methods}

Chicken eggs, incubation and housing. Specific pathogen free (SPF) eggs that were obtained from the Animal Disease Research Institute, Canadian Food Inspection Agency (Ottawa, Ontario, Canada) were incubated at recommended temperature and relative humidity at the Arkell Research Station, University of Guelph. All experiments were approved by the Animal Care Committee of the University of Guelph and conducted according to the guidelines of the Animal Care Committee of the University of Guelph. At ED18, live embryos were administrated with ECpG or HVT or both into the amniotic sac using a 23-gauge, $2.5 \mathrm{~cm}$ needle as recommended. Once the chicks hatched, they were transported to the animal isolation facility at the Ontario Veterinary College, University of Guelph.

TLR ligand, vaccine and virus. Synthetic Class B CpG ODN 2007 [5'-TCGTCGTTGTCGTTTTGTCGTT-3'] with phosphorothioate backbone was purchased from Sigma-Aldrich (Oakville, ON, Canada). FC-126 strain of HVT vaccine was received from Merial Canada Inc (Boehringer-Ingelheim Canada Ltd, Quebec, Canada). In vivo propagated, very virulent strain of MDV (RB1B) titrated in chicken kidney cells was used to infect chicks ${ }^{48}$.

Encapsulation of TLR ligand. CpG ODN was encapsulated in PLGA, (Resomer ${ }^{\circledR}$ RG 503 H, free carboxylic acid, MW 24-38 kD, Sigma-Aldrich) using the modified double emulsion solvent evaporation method to generate PLGA nanoparticles as described in our previous studies ${ }^{19,28}$. Briefly, polyethalenimine (linear, MW $2.5 \mathrm{kD}$, Sigma-Aldrich) was complexed with $\mathrm{CpG}$ at a defined ratio ${ }^{49}$. This complex was layered onto $4.5 \%$ PLGA dissolved in dichloromethane (DCM, Sigma-Aldrich) and sonicated for one minute at $40 \%$ amplitude using an Ultrasonic Processor (Fisher Scientific). The resulting emulsion was sonicated with $2 \%$ polyvinyl alcohol (PVA)/1\% poloxamer (PVA, MW 30-70 kD, 87-90\% hydrolyzed, Sigma Aldrich) for two minutes at $60 \%$ amplitude and the resulting suspension was hardened by mixing with $50 \mathrm{~mL}$ of $2 \% \mathrm{PVA} / 1 \%$ poloxamer solution. The PLGA nanoparticles were harvested by centrifugation at $20,000 \mathrm{~g}$ for $50 \mathrm{~min}$ at $4{ }^{\circ} \mathrm{C}$ and washed three times with ultrapure nuclease free water. The PLGA nanoparticle suspension was frozen at $-80^{\circ} \mathrm{C}$ for $1-2$ hours, 


\begin{tabular}{|c|c|c|}
\hline Genes & Primer sequences & References \\
\hline \multirow{2}{*}{$\beta$-actin } & F: 5'-CAACACAGTGCTGTCTGGTGGTA-3' & \multirow[t]{2}{*}{52} \\
\hline & R: 5'-ATCGTACTCCTGCTTGCTGATCC-3' & \\
\hline \multirow{2}{*}{ meq } & F: 5'-GTCCCCCCTCGATCTTTCTC-3' & \multirow{2}{*}{50} \\
\hline & R: 5'-CGTCTGCTTCCTGCGTCTTC-3?' & \\
\hline \multirow{2}{*}{ IFN- $\beta$} & F: 5'-GCCTCCAGCTCCTTCAGAATACG-3' & \multirow{2}{*}{53} \\
\hline & R: 5'-CTGGATCTGGTTGAGGAGGCTGT-3' & \\
\hline \multirow{2}{*}{ IFN- $\gamma$} & F: $5^{\prime}$-ACACTGACAAGTCAAAGCCGC-3' & \multirow{2}{*}{54} \\
\hline & R: 5'-AGTCGTTCATCGGGAGCTTG-3' & \\
\hline \multirow{2}{*}{ IL-18 } & F: 5'-GAAACGTCAATAGCCAGTTGC-3' & \multirow{2}{*}{55} \\
\hline & R: 5'-TCCCATGCTCTTTCTCACAACA-3' & \\
\hline \multirow{2}{*}{ IL-1 $\beta$} & F: 5'-GTGAGGCTCAACATTGCGCTGTA-3' & \multirow{2}{*}{56} \\
\hline & R: 5'-TGTCCAGGCGGTAGAAGATGAAG-3' & \\
\hline \multirow{2}{*}{ IL-10 } & F: 5'-AGCAGATCAAGGAGACGTTC-3' & \multirow{2}{*}{41} \\
\hline & R: 5'ATCAGCAGGTACTCCTCGAT-3' $^{\prime}$ & \\
\hline
\end{tabular}

Table 1. Target genes, primer sequences and references used for real-time PCR.

then lyophilized for 18-22 hours (FreeZone ${ }^{\circledR} 18$ Liter Freeze Dry Systems, Labconco Corporation, Kansas City, Missouri) and sterilized as indicated ${ }^{28}$. Encapsulation efficiency was determined as in our previous studies ${ }^{19,28}$.

Experimental design. Seven groups of ED18 chicken embryos (38-45 embryos per group) were injected via the amniotic route with approximately $25 \mu \mathrm{g}$ ECpG and a quarter (1/4) of the recommended dose of HVT or HVT alone or phosphate buffered saline (PBS). Once the chicks hatched, at 5 days of age (5d), all chicks, except the PBS group were infected with 250 plaque-forming unit (PFU) of very virulent RB1B MDV per chick via intra-abdominal route. The untreated, but MDV-infected group was used as a positive control group. At $14 \mathrm{dpi}$, chickens were given a second dose of ECPG or PBS. Experimental groups in this study were designated as follows. Chickens in group 1 (G1) received ECpG and HVT at ED18, infected with MDV at day 5 (d5) and received a second dose of ECpG at 14 dpi. Group 2 (G2) chickens were administered with ECpG and HVT at ED18 and infected with MDV at d5. Chickens in group 3 (G3) were injected with HVT at ED18, infected with MDV at d5 and administered ECpG at 14 dpi. Group 4 (G4) chickens were administered with HVT at ED18 and infected with MDV at d5. Group 5 (G5) chickens received MDV at d5 and ECpG at 14 dpi. Group 6 (G6) chickens received MDV at d5. Chickens in group 7 (G7) received PBS. Each group had between 9-15 chickens at different time points. At 4, 10 and $21 \mathrm{dpi}$, spleen, and bursa of Fabricius weight (BF), and body weight (BW) were recorded and organ weight indices were calculated relative to the body weight of chickens. Samples from spleen, BF and feathers were collected in RNAlater and stored at $-80^{\circ} \mathrm{C}$ until further processing. Tumor incidence following infection with RB1B MDV was documented at $21 \mathrm{dpi}$.

DNA and RNA extraction and cDNA synthesis. Genomic DNA was extracted from feather tips as described previously ${ }^{50}$. Feather tips were cut into small pieces and incubated with $500 \mu \mathrm{l}$ cell lysis buffer $(10 \mathrm{mM}$ Tris, $\mathrm{pH} 7.5,10 \mathrm{mM} \mathrm{NaCl}, 1 \mathrm{mM}$ EDTA, $\mathrm{pH} 8$ with $0.5 \%$ (w/v) Sarkosyl) containing $100 \mu$ of proteinase K (10 mg/ $\mathrm{ml}$ ) overnight at $65^{\circ} \mathrm{C}$ water bath. Extracted DNA was precipitated using $25 \mu \mathrm{l} \mathrm{M} \mathrm{NaCl}$ and $2.3 \mathrm{ml} 95 \%$ ethanol. DNA concentration was measured and dilutions at a concentration of $50 \mathrm{ng} / \mu \mathrm{l}$ of DNA samples were prepared. A hundred nanograms of diluted DNA was used for MDV genome copy numbers quantification using quantitative real-time PCR.

RNA was extracted from spleen and BF using Trizol (Life Technologies, Burlington, Ontario) according to the manufacturer's protocol. cDNA was synthesized from $1 \mu \mathrm{g}$ of DNase treated RNA using Oligo $\mathrm{d}_{(\mathrm{dT})} 12-18$ primers and the Super-Script ${ }^{\mathrm{TM}}$ First-Strand Synthesis System kit (Life Technologies, Burlington, Canada) according to manufacturer's instructions. Synthesized cDNA was diluted at 1:10 in nuclease free water for evaluating the expression of cytokine genes. The quantity and quality of DNA and RNA were determined using the NanoDrop ${ }^{\circledR}$ ND-1000 spectrophotometry (NanoDrop Technologies, Wilmington, DE).

Real-time PCR. Real-time PCR was performed using SYBR green dye in a LightCycler 480 II to quantify the MDV genome copy numbers and cytokine gene expression (Roche Diagnostics, Laval, Quebec) as described previously ${ }^{50,51}$. Primer sequences of target and reference genes are listed in Table 1 . The primers were synthesized by Sigma-Aldrich Canada (Oakville, ON).

Statistical analysis. Relative target gene expression was calculated using chicken $\beta$-actin in LightCycler 480 II advanced relative quantification software. Data was logarithmically transformed and analyzed using general linear model (Proc GLM) in Statistical Analysis Software version 9.3 (SAS, Cary, NC). Duncan's multiple range test was performed to determine significantly different groups. Gene expression results were presented as the geometric mean of relative expression \pm standard error of mean. Tumor incidence data were analyzed by Fisher's exact test (GraphPad Prism version 6.04). One-way ANOVA followed by Tukey's multiple comparison test was used to analyze BF:BW and spleen:BW ratios, and MDV genome copy numbers. When data were not normally distributed Kruskal-Wallis test was used. Results were considered significant if $p$ value was $<0.05$. 


\section{Data Availability Statement}

The datasets generated and analyzed during the current study are available from the corresponding author on reasonable request.

\section{References}

1. Osterrieder, N., Kamil, J. P., Schumacher, D., Tischer, B. K. \& Trapp, S. Marek's disease virus: from miasma to model. Nat. Rev. Microbiol. 4, 283-294 (2006).

2. Baigent, S. J. \& Davison, F. In Marek's Disease: An Evolving Problem (eds Davison, F. \& Nair, V.) 62-77 (Elsevier Academic Press, 2004).

3. Calnek, B. W. Lymphomagenesis in Marek's disease. Avian Pathol. 27, 54-64 (1998).

4. Gurung, A., Kamble, N., Kaufer, B. B., Pathan, A. \& Behboudi, S. Association of Marek's Disease induced immunosuppression with activation of a novel regulatory T cells in chickens. PLOS Pathog. 13, e1006745 (2017).

5. Nair, V. Evolution of Marek's disease - A paradigm for incessant race between the pathogen and the host. Vet. J. 170, 175-183 (2005).

6. Bublot, M. \& Sharma, J. In Marek's Disease: An Evolving Problem (eds Davison, F. \& Nair, V.) 168-185 (Elsevier Academic Press, 2004).

7. Witter, R. L. Increased Virulence of Marek's Disease Virus Field Isolates. Avian Dis. 41, 149-163 (1997).

8. Haq, K., Schat, K. A. \& Sharif, S. Immunity to Marek's disease: where are we now? Dev. Comp. Immunol. 41, 439-446 (2013).

9. Schijns, V. E. J. C., van de Zande, S., Lupiani, B. \& Reddy, S. M. In Avian Immunology: Second Edition (eds Schat, K. A., Kaspers, B. \& Kaiser, P.) 345-362 (Elsevier Ltd, 2013).

10. Abdul-Cader, M. S. et al. Hatchery Vaccination Against Poultry Viral Diseases: Potential Mechanisms and Limitations. Viral Immunol. 31, 23-33 (2018).

11. Negash, T., Al-Garib, S. O. \& Gruys, E. Comparison of in ovo and post-hatch vaccination with particular reference to infectious bursal disease. A review. Vet. Q. 26, 76-87 (2004).

12. Lowenthal, J. W., Connick, T., McWaters, P. G. \& York, J. J. Development of T cell immune responsiveness in the chicken. Immunol. Cell Biol. 72, 115-122 (1994).

13. Mast, J. \& Goddeeris, B. M. Development of immunocompetence of broiler chickens. Vet. Immunol. Immunopathol. 70, 245-256 (1999).

14. Van Ginkel, F. W. et al. Age-dependent immune responses and immune protection after avian coronavirus vaccination. Vaccine 33, 2655-2661 (2015).

15. Abdul-Careem, M. F., Hunter, D. B., Lambourne, M. D., Barta, J. \& Sharif, S. Ontogeny of Cytokine Gene Expression in the Chicken Spleen. Poult. Sci. 86, 1351-1355 (2007).

16. Abdul-Careem, F. et al. Expression of cytokine genes following pre- and post-hatch immunization of chickens with herpesvirus of turkeys. Vaccine 26, 2369-2377 (2008).

17. Heller, E. D. \& Schat, K. A. Enhancement of natural killer cell activity by Marek's disease vaccines. Avian Pathol. 16, 51-60 (1987).

18. Quere, P., Dambrine, G. V. C. A. D. R. \& Dambrine, G. Development of anti-tumoral cell-mediated cytotoxicity during the course of Marek's disease in chickens. Ann Rech V'et 19, 193-201 (1988).

19. Bavananthasivam, J., Alkie, T. N., Matsuyama-Kato, A., Hodgins, D. C. \& Sharif, S. Characterization of innate responses induced by in ovo administration of encapsulated and free forms of ligands of Toll-like receptor 4 and 21 in chicken embryos. Res. Vet. Sci., 0-1 (2017).

20. Gimeno, I. M. et al. In Ovo Vaccination with Turkey Herpesvirus Hastens Maturation of Chicken Embryo Immune Responses in Specific-Pathogen-Free Chickens. Avian Dis. 59, 375-83 (2015).

21. Mallick, A. I. et al. Vaccination with CpG-adjuvanted avian influenza virosomes promotes antiviral immune responses and reduces virus shedding in chickens. Viral Immunol. 25, 226-31 (2012).

22. St. Paul, M. et al. Effects of Ligands for Toll-Like Receptors 3, 4, and 21 as Adjuvants on the Immunogenicity of an Avian Influenza Vaccine in Chickens. Viral Immunol. 27, 167-173 (2014).

23. St. Paul, M. et al. Prophylactic treatment with Toll-like receptor ligands enhances host immunity to avian influenza virus in chickens. Vaccine 30, 4524-4531 (2012).

24. Barjesteh, N. et al. Reduction of avian influenza virus shedding by administration of Toll-like receptor ligands to chickens. Vaccine 33, 4843-4849 (2015).

25. Parvizi, P., Mallick, A. I., Haq, K., Schlegel, B. \& Sharif, S. A. Toll-like receptor 3 agonist (polyI:C) elicits innate host responses in the spleen and lungs of chickens. Can. J. Vet. Res. 76, 230-234 (2012).

26. Parvizi, P. et al. A Toll-Like Receptor 3 Ligand Enhances Protective Effects of Vaccination Against Marek's Disease Virus and Hinders Tumor Development in Chickens. Viral Immunol. 25, 394-401 (2012).

27. Parvizi, P. et al. The effects of administration of ligands for Toll-like receptor 4 and 21 against Marek's disease in chickens. Vaccine 32, 1932-1938 (2014).

28. Alkie, T. N. et al. Characterization of Innate Responses Induced by PLGA Encapsulated- and Soluble TLR Ligands In Vitro and In Vivo in Chickens. PLoS One 12, (2017).

29. Bavananthasivam, J. et al. In ovo administration of Toll-like receptor ligands encapsulated in PLGA nanoparticles impede tumor development in chickens infected with Marek's disease virus. Vaccine 36, 4070-4076 (2018).

30. Haq, K., Fear, T., Ibraheem, A., Abdul-Careem, M. F. \& Sharif, S. Influence of vaccination with CVI988/Rispens on load and replication of a very virulent Marek's disease virus strain in feathers of chickens. Avian Pathol. 41, 69-75 (2012).

31. De Boer, G. F., Groenendal, J. E., Boerrigter, H. M., Kok, G. L. \& Pol, J. M. A. Protective Efficacy of Marek's Disease Virus (MDV) CVI-988 CEF 65 Clone C against Challenge Infection with Three Very Virulent MDV Strains. Source Avian Dis. 30, 276-283 (1986).

32. Hunt, H. D. \& Dunn, J. R. The Influence of Major Histocompatibility Complex and Vaccination with Turkey Herpesvirus on Marek's Disease Virus Evolution. Avian Dis. 59, 122-9 (2015).

33. Islam, A. F. M. F. et al. Immunosuppressive effects of Marek's disease virus (MDV) and herpesvirus of turkeys (HVT) in broiler chickens and the protective effect of HVT vaccination against MDV challenge. Avian Pathol. 31, 449-461 (2002).

34. Witter, R. L., Calnek, B. W., Buscaglia, C., Gimeno, I. M. \& Schat, K. A. Classification of Marek's disease viruses according to pathotype: Philosophy and methodology. Avian Pathol. 34, 75-90 (2005).

35. Pierce, A. T. et al. Beta interferon and gamma interferon synergize to block viral DNA and virion synthesis in herpes simplex virusinfected cells. J. Gen. Virol. 86, 2421-2432 (2005).

36. Gill, N., Deacon, P. M., Lichty, B., Mossman, K. L. \& Ashkar, A. A. Induction of innate immunity against herpes simplex virus type 2 infection via local delivery of Toll-like receptor ligands correlates with beta interferon production. J. Virol. 80, 9943-50 (2006).

37. Djeraba, A., Bernardet, N., Dambrine, G. \& Quéré, P. Nitric oxide inhibits Marek's disease virus replication but is not the single decisive factor in interferon-gamma-mediated viral inhibition. Virology 277, 58-65 (2000).

38. Haq, K. et al. Effects of interferon- g knockdown on vaccine-induced immunity against Marek' s disease in chickens. Can. J. Vet. Res. 1, 1-7 (2015).

39. Haq, K. et al. Interferon- $\gamma$ influences immunity elicited by vaccines against very virulent Marek's disease virus. Antiviral Res. 90, 218-226 (2011). 
40. Rothwell, L. et al. Cloning and Characterization of Chicken IL-10 and Its Role in the Immune Response to Eimeria maxima. J. Immunol. 173, 2675-2682 (2004).

41. Abdul-Careem, M. F. et al. Cytokine gene expression patterns associated with immunization against Marek's disease in chickens. Vaccine 25, 424-432 (2007).

42. Parvizi, P. et al. Cytokine gene expression in splenic CD4+ and CD8+ T cell subsets of genetically resistant and susceptible chickens infected with Marek's disease virus. Vet. Immunol. Immunopathol. 132, 209-217 (2009).

43. Steinbrink, K., Wölfl, M., Jonuleit, H., Knop, J. \& Enk, A. H. Induction of tolerance by IL-10-treated dendritic cells. J. Immunol. 159, $4772-80$ (1997)

44. Steinbrink, K. et al. Interleukin-10-treated human dendritic cells induce a melanoma-antigen-specific anergy in CD8(+) T cells resulting in a failure to lyse tumor cells. Blood 93, 1634-42 (1999).

45. Hsu, P. et al. IL-10 Potentiates Differentiation of Human Induced Regulatory T Cells via STAT3 and Foxo1. J. Immunol. 195, 3665-3674 (2015).

46. Kaiser, P., Underwood, G. \& Davison, F. Differential Cytokine Responses following Marek' s Disease Virus Infection of Chickens Differing in Resistance to Marek's Disease. J. Virol. 77, 762-768 (2003).

47. Vidal-Vanaclocha, F. et al. IL-18 regulates IL-1beta-dependent hepatic melanoma metastasis via vascular cell adhesion molecule-1. Proc. Natl. Acad. Sci. USA 97, 734-739 (2000).

48. Schat, K. A., Calnek, B. W. \& Fabricant, J. Characterisation of two Highly Oncogenic Strains of Marek's Disease Virus. Avian Pathol. 11, 593-605 (1982).

49. Boussif, O. et al. A versatile vector for gene and oligonucleotide transfer into cells in culture and in vivo: polyethylenimine. Proc. Natl. Acad. Sci. USA 92, 7297-301 (1995).

50. Abdul-Careem, M. F. et al. Development of a real-time PCR assay using SYBR Green chemistry for monitoring Marek's disease virus genome load in feather tips. J. Virol. Methods 133, 34-40 (2006).

51. St. Paul, M. et al. In vivo administration of ligands for chicken toll-like receptors 4 and 21 induces the expression of immune system genes in the spleen. Vet. Immunol. Immunopathol. 144, 228-237 (2011).

52. Brisbin, J. T. et al. Gene expression profiling of chicken lymphoid cells after treatment with Lactobacillus acidophilus cellular components. Dev. Comp. Immunol. 32, 563-574 (2008).

53. Abdul-Careem, F. et al. Host responses in the bursa of Fabricius of chickens infected with virulent Marek's disease virus. Virology 379, 256-265 (2008).

54. Brisbin, J. T., Gong, J., Parvizi, P. \& Sharif, S. Effects of lactobacilli on cytokine expression by chicken spleen and cecal tonsil cells. Clin. Vaccine Immunol. 17, 1337-1343 (2010).

55. Abdul-Careem, M. F. et al. Marek's disease virus-induced transient paralysis is associated with cytokine gene expression in the nervous system. Viral Immunol. 19, 167-176 (2006).

56. Abdul-Careem, F. et al. Induction of innate host responses in the lungs of chickens following infection with a very virulent strain of Marek's disease virus. Virology 393, 250-257 (2009).

\section{Acknowledgements}

Jegarubee Bavananthasivam is a recipient of an Ontario Trillium Scholarship. This work was funded by the Natural Sciences and Engineering Research Council of Canada, and the Ontario Ministry of Agriculture, Food and Rural Affairs. We would like to thank the staff of the isolation facility of Ontario Veterinary College for housing and caring of the chickens and Arkell Poultry Research Station of University of Guelph for incubating the eggs.

\section{Author Contributions}

Conceived and designed the experiments: J.B., S.S. Performed the experiments: J.B., L.R., J.A., A.Y. Analyzed the data: J.B. Contributed to writing and reviewing of the manuscript: J.B., L.R., J.A., A.Y., T.N.A., M.F.A., S.K.W., S.B., S.S

\section{Additional Information}

Supplementary information accompanies this paper at https://doi.org/10.1038/s41598-018-34760-6.

Competing Interests: The authors declare no competing interests.

Publisher's note: Springer Nature remains neutral with regard to jurisdictional claims in published maps and institutional affiliations.

(1) Open Access This article is licensed under a Creative Commons Attribution 4.0 International c. License, which permits use, sharing, adaptation, distribution and reproduction in any medium or format, as long as you give appropriate credit to the original author(s) and the source, provide a link to the Creative Commons license, and indicate if changes were made. The images or other third party material in this article are included in the article's Creative Commons license, unless indicated otherwise in a credit line to the material. If material is not included in the article's Creative Commons license and your intended use is not permitted by statutory regulation or exceeds the permitted use, you will need to obtain permission directly from the copyright holder. To view a copy of this license, visit http://creativecommons.org/licenses/by/4.0/.

(C) The Author(s) 2018 